\title{
Description of Allothrombium triticium adult (Acari: Trombidiidae) from Iran
}

\author{
ALIREZA SABOORI ${ }^{1} \&$ KARIM KAMALI ${ }^{2}$ \\ ${ }^{1}$ Department of Plant Protection, College of Agriculture, Tehran University, Karaj, Iran. E-mail:saboori@af.ut.ac.ir \\ ${ }^{2}$ Department of Entomology, College of Agriculture, Tarbiat Modarres University, Tehran, Iran
}

\section{Introduction}

Allothrombium triticium Zhang, 1995 (Acari: Trombidiidae) was described from larvae ectoparasitic on aphids in Shahrkord, Iran (Zhang \& Norbakhsh 1995). In this note, we describe the adult female of this species from specimens collected under stones in Tehran, Iran.

\section{Material and methods}

Some adults trombidiid mites were collected under stones on 29 November 1996 in Tehran, Iran. They were reared in a laboratory at $25 \pm 1^{\circ} \mathrm{C}, 70-80 \% \mathrm{RH}$ and $15 \mathrm{~L} / 9 \mathrm{D}$ in a plastic petri dish (diameter $15 \mathrm{~cm}$ and height $4 \mathrm{~cm}$ ) with moist soil as the substrate and were fed with rose aphids (Macrosiphum rosae (L.)). When eggs were laid, one egg mass was placed in a vial (diameter $2.5 \mathrm{~cm}$ and length 6 $\mathrm{cm}$ ) plugged with a ball of cotton to prevent larvae from escaping. The vial with the egg mass was held at $25 \pm 1{ }^{\circ} \mathrm{C}, 80-95 \% \mathrm{RH}$ and $15 \mathrm{~L} / 9 \mathrm{D}$. When larvae appeared, they were determined as Allothrombium triticium. Some adult females and larvae were cleared in Nesbitt's fluid and mounted on slides using Hoyer's medium (Krantz 1978).

All measurements, given in micrometers, are in the format: average (min.-max.). Abbreviations and terminology used in this note are adapted from Southcott (1986). Specimens are deposited in the Acarological Collection, Zoological Museum, College of Agriculture, Tehran University, Karaj, Iran.

\section{Descriptions}

Egg: Colour in life orange. Spheroidal in shape. Diameter 247-357 (in the adult body).

Female (Figs.1-4): From a slide (ARS-19961129-1b), Iran, Tehran, A.Saboori, 29 November 1996. Idiosoma 3850 (3657-3988) long to posterior end of chelicerae, 4640 (4509-4730) to tip of chelicerae, maximum width 2186 (2062-2310), covered with setae. Crista and lateral plates separate and distinct (Fig.1). Crista metopica with some long setulose setae anteriorly. Sensillae long, filiform, with distal half setulose. Two lateral plates large, each with some long setulose setae. A pair of eyes on a pair of peduncles; anterior eye larger than posterior ones. Measurements: ASB 310 (278332), PSB 256 (192-299), L 566 (470- 631), W 205 (192-223), SB 136 (128-150), Sens 268 (247289), EPL 163 (135-176), EPW 144 (143-146),MDS 82-113 (50-121), PDS 167 (165-176).

External genitalia $441(428-471) \times 276(215-375)$, with $3+3$ acetabulae (Fig.2); internal and external valves with some setae. Anus $125(43-160) \times 36(32-75)$. 

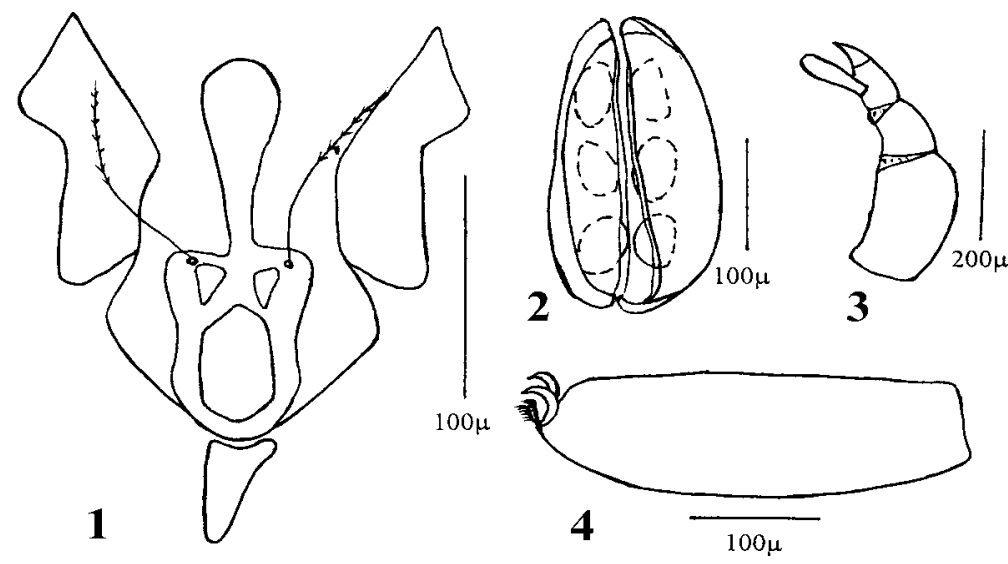

FIGURES 1-4. Allothrombium triticium Zhang (adult, female). 1, crista; 2, external genitalia; 3, palp; 4, tarsus I.

Palpi normal. Palptarsus clavate, 228 (214-235) $\times 67$ (64-74) (Fig.3). Chelicerae robust, basis elongate-pyriform. Cheliceral base 599 (588-620) long, 163 (139-203) wide. Cheliceral blades robust, 161 (150-171) long, with 12 dorsal denticles.

Legs covered with short setae. Leg I longer than other legs; leg III shortest. Leg length including coxae and excluding claws: I 3254 (2887-3575), II 2220 (2145-2337), III 2046 (2035-2067), IV 2952 (2722-3245). Each tarsus with a pair of claws and feathered empodium (Fig.4). Measurments of leg segments: TaI (L) 542 (525-567), TaI (H) 162 (160-165), TiI 651 (642-663), GeI 587 (585-588), TaII (L) 397 (385-412), TaII (H) 114 (96-139), TiII 386 (357-406), GeII 348 (330-363), TaIII (L) 352 (342-357), TaIII (H) 112 (110-117), TiIII 365 (353-385), GeIII 341 (330-350), TaIV(L) 442 (438450), TaIV (H) 134 (118-150), TiIV 570 (525- 588), GeIV 485 (440-503).

\section{Acknowledgements}

We thank Prof. Z.-Q. Zhang (Landcare Research, New Zealand) for a critical review of the manuscript. The project on which this paper was based was supported by a grant from the Zoological Museum, College of Agriculture, Tehran University, Karaj, Iran. The senior author's space and facilities during this study were kindly provided by the Department of Entomology , College of Agriculture, Tarbiat Modarres University, Tehran, Iran.

\section{References}

Krantz, G.W. (1978) A Manual of Acarology. 2nd Edition, Corvallis, Oregon, Oregon State University Book Store, $508 \mathrm{pp}$.

Southcott, R.V. (1986) Studies on the taxonomy and biology of the subfamily Trombidiinae (Acarina:Trombidiidae) with a critical revision of the genera. Australian Journal of Zoology (Supplementary Series), 123, 1-116.

Zhang, Z.-Q. \& Norbakhsh, H. (1995) A new genus and three new species of mites (Acari: Trombidiidae) described from larvae ectoparasitic on aphids from Iran. European Journal of Entomology, 92, 705-718.

Accepted: 6 March 2000 